\title{
Improvement in Hydrogen Sensing Response of Zinc Oxide Doped with Platinum
}

\author{
Anita Hastir', Nipin Kohli ${ }^{2}$, Ravi Chand Singh ${ }^{1}$ \\ ${ }^{1}$ Department of Physics, Guru Nanak Dev University \\ Amritsar-143005, India \\ ${ }^{2}$ Department of Physics, BBK DAV College for Women \\ Amritsar-143001, India \\ nipinkohli82@yahoo.com
}

\begin{abstract}
In this work, the effect of Platinum $(\mathrm{Pt})$ as dopant on the structural, morphological and gas sensing properties of $\mathrm{ZnO}$ has been discussed. $\mathrm{ZnO}$ and Ptdoped $\mathrm{ZnO}$ nanoparticles were synthesized by facile and cost effective co-precipitation technique. XRD analysis revealed the formation of hexagonal wurtzite structure for pure and doped nanostructures which was further supported by Raman studies.Raman and X-Ray photoelectron spectroscopy (XPS) investigations also reveal the presence of defects in doped samples. The morphology of the synthesised samples has been studied by field emission scanning electron microscopy (FESEM). For gas sensing characteristics the synthesized particles were applied as thick film onto an alumina substrate and tested at different operating temperatures for hydrogen gas. Among all samples, $0.05 \% \mathrm{Pt}$ doped $\mathrm{ZnO}$ exhibited enhanced sensing performance towardshydrogen. The increase in sensing response is attributed to presence of defects in doped sample and the catalytic nature of platinum.
\end{abstract}

Keywords: Nanoparticles, Pt- doped ZnO, Hydrogen Sensor, Catalyst

\section{Introduction}

Hydrogen gas is extensively used as a clean fuel for space applications, industrial processes, metallurgical processes, nuclear reactors, power generation, etc. [1]. Being highly inflammable its usage at concentrations in excess of $4 \%$ vol. in ambient air can lead to explosion risks. Since it is a colourless and odourless gas therefore its leakage detection by human senses is not possible [2,3]. Thus the hydrogen sensor is of utmost importance in order to detect its early leakage. Semiconductor metal oxides (SMOs) have been extensively used in the application of gas sensing because of the low cost, high sensitivity and low power consumption. Among these oxides, $\mathrm{ZnO}$, n-type SMO intrinsically has gained interest of scientific fraternity due to its striking physical and chemical properties $[4,5]$.

Various factors such as grain size, morphology, surface to volume ratio and defects are responsible for variation in sensing properties of a sensor material. These properties can be modified either by addition of dopant or using different synthesis method in order to improve the sensor properties.

In the present work the doped $\mathrm{ZnO}$ samples has been used as a hydrogen sensor with a significant enhancement in $\mathrm{H}_{2}$ gas sensing response, reduction in optimum operable temperature and fast response /recovery of the fabricated sensor.

\section{Experimental}

\subsection{Synthesis and material characterizations}

All reagents used were of analytically grade purchased from Sigma Aldrich. 0.2M aqueous solution of zinc acetate dihydrate was prepared with continuous stirring at room temperature. To the above aqueous solution ammonium hydroxide was added slowly till the required $\mathrm{pH} 8$ was attained. The precipitates thus obtained were filtered, washed several times with distilled water and then dried in oven at $120^{\circ} \mathrm{C}$. The dried samples were ground and calcined at $500^{\circ} \mathrm{C}$ for 3 hours. Similarly, $\mathrm{Pt}$-doped $\mathrm{ZnO}$ samples were prepared by adding platinum (IV) chloride $(0.02,0.05$, and $0.2 \mathrm{wt}$. \%) into aqueous solution of zinc acetate dihydrate and the rest of the procedure is same as above.

The X-ray diffraction studies were carried out using $\mathrm{Cu}-\mathrm{K} \alpha$ radiation with Shimadzu 7000 Diffractometer in order to investigate the crystalline phases present in the synthesized samples. The morphology of the samples were analysed by 
field emission scanning electron microscope with Carl Zeiss Supra 55. Raman investigations were done at room temperature using Renishaw inVia Micro Raman spectrometer with an argon-ion laser at an excitation wavelength of $488 \mathrm{~nm}$. X-ray photoelectron spectroscopy measurements were performed in a PHI-5600 instrument.

\subsection{Sensor Fabrication and Gas sensing apparatus}

Sensing properties of the samples was measured in a home-made apparatus consisting of a potentiometric arrangement reported elsewhere [6] and the sensing response for ethanol was recorded by Keithley Data Acquisition Card KUSB-3100. The sensor response magnitude was determined as $\mathrm{Ra} / \mathrm{Rg}$ ratio, where $\mathrm{Ra}$ and $\mathrm{Rg}$ were the resistances of sensor in air and air-gas ambience respectively.

\section{Results and Discussion}

\subsection{XRD Analysis}

Fig. 1 represents the X-ray diffraction pattern of pure and Pt-doped $\mathrm{ZnO}$ samples. The peak positions confirm the hexagonal wurtzite structure of synthesised samples. No peaks corresponding to Pt metal or platinum oxide are present which implies that dopant is successfully incorporated in $\mathrm{ZnO}$ lattice. A shift in peaks towards higher theta values is observed with increase in dopant content which is attributed to intrusion of Pt having smaller ionic radii $(0.62 \AA)$ as compared to $\mathrm{Zn}(0.74$ $\AA$ ) [7]. The crystallite size calculated using Scherrer's formula was found to be $16.06,16.35$ and $17.15 \mathrm{~nm}$ for $0.02,0.05$ and $0.2 \%$ Pt-doped $\mathrm{ZnO}$ nanoparticles respectively.

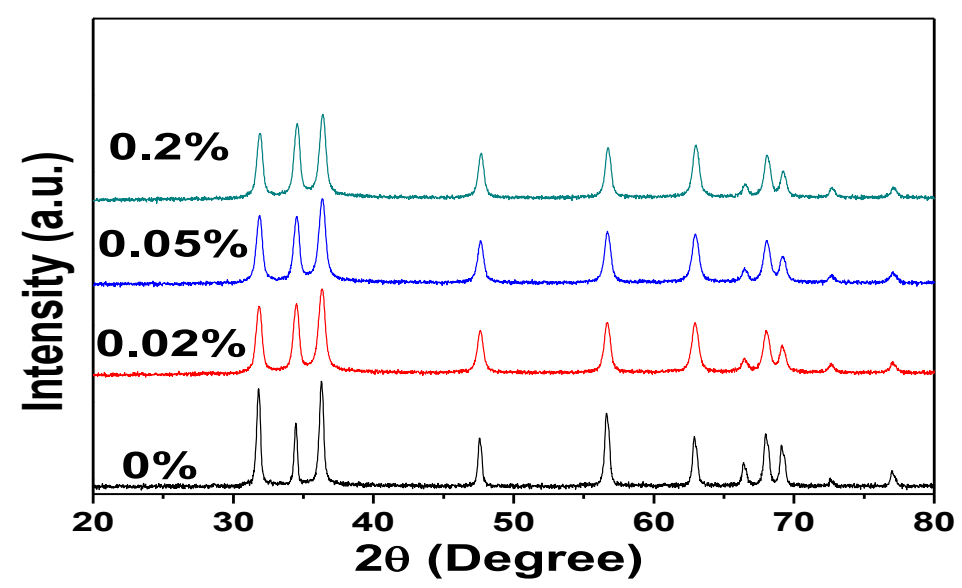

Fig. 1: XRD patterns of pure and $\mathrm{Pt}$-doped $\mathrm{ZnO}$ nanostructures.

\subsection{Raman Spectroscopy}

A room temperature Raman study has been carried out in order to find the structural and vibrational properties of pure and doped $\mathrm{ZnO}$. Raman-shift data from 200 to $800 \mathrm{~cm}^{-1}$ for undoped and doped $\mathrm{ZnO}$ is shown in Fig. 2. Pure $\mathrm{ZnO}$ Raman peaks are observed at $333,380,438,581 \mathrm{~cm}^{-1}$. The $438 \mathrm{~cm}^{-1}$ is the characteristics peak corresponding to hexagonal wurtzite structure of zinc oxide. This peak became broader and less intense with increase in dopant concentration implying the loss in crystallinity whereas the intensity of Raman mode around $581 \mathrm{~cm}^{-1}$ increases in doped samples indicating the increase in defects such as oxygen vacancies with dopant introduction. A shift towards lower frequency values is observed with dopant addition which could be related to reduction in particle size lying in nano-meter regime as explained by phonon confinement model $[8,9]$. 


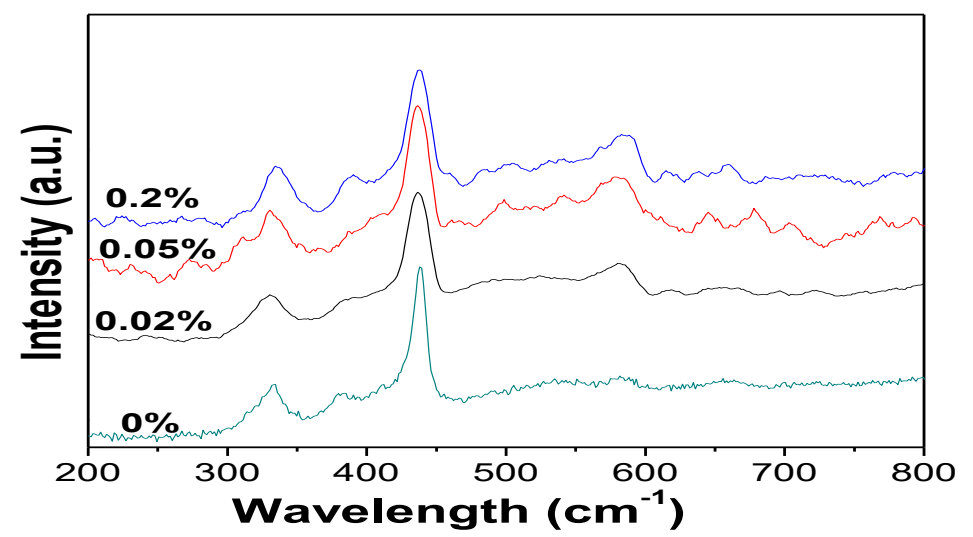

Fig. 2: Raman Spectra of pure and Pt- doped $\mathrm{ZnO}$ samples.

\subsection{FESEM Analysis}

To know the morphology of the synthesised samples FESEM was performed and the micrographs for pure and $0.05 \%$ Pt doped sample are shown in Fig. 3(a) and (b). For pure $\mathrm{ZnO}$, dumbbell shaped micro rods are formed while doped samples have nearly spherical shaped particles having dimensions in nano-meter scale. FESEM measurements clearly showed the change in morphology and decrease in particle size with introduction of dopant.

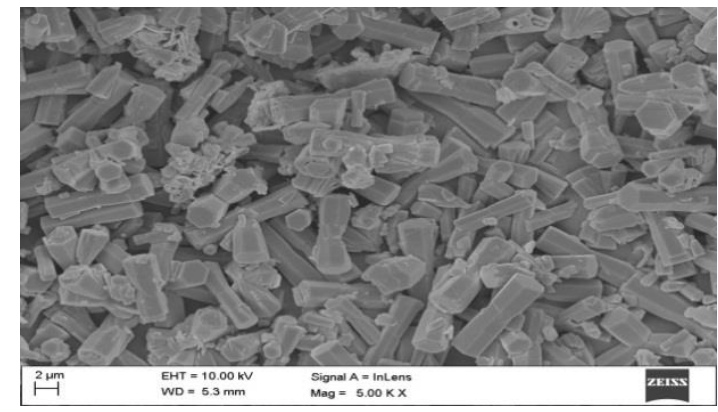

(a)

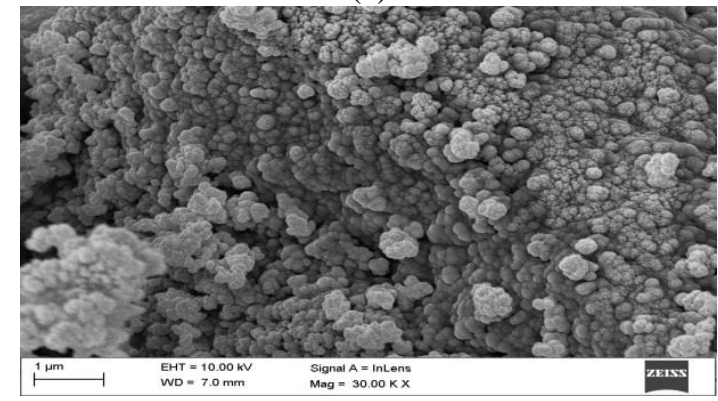

(b)

Fig. 3: FESEM image of (a) pure, (b) $0.05 \%$ Pt dopedZnO.

\subsection{XPS}

Figure 4 (a) and (b) shows Oxygen 1s high resolution XPS spectra of pure and $0.05 \%$ Pt doped $\mathrm{ZnO}$. The O1s peak of pure $\mathrm{ZnO}$ deconvolutes into three peaks: O1 (B.E. $530.74 \mathrm{eV}$ ) associated to lattice oxygen forming bonds with lattice cations, $\mathrm{O} 2$ (B.E. $531.83 \mathrm{eV}$ ) ascribed to oxygen deficient regions and O3(B.E. $532.48 \mathrm{eV}$ ) related to chemisorbed oxygen[10]. However the O1s peak of $0.05 \%$ Pt-doped $\mathrm{ZnO}$ is Gaussian divided into three peaks having B.E.530.08, 530.65, $532.07 \mathrm{eV}$ respectively. Fig 4(a) and (b) clearly shows that the intensity of oxygen deficient peak increases in $0.05 \% \mathrm{Pt}$ doped $\mathrm{ZnO}$ implying increase in oxygen defects in doped sample. 


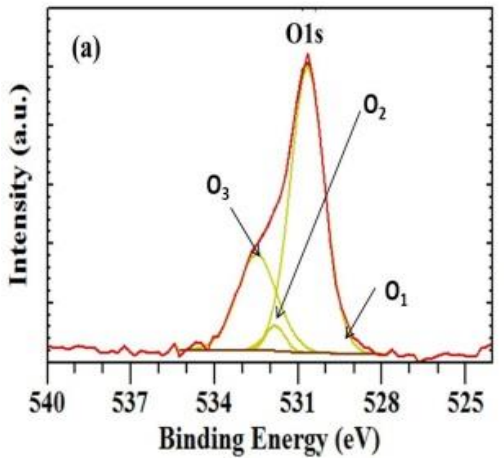

(a)

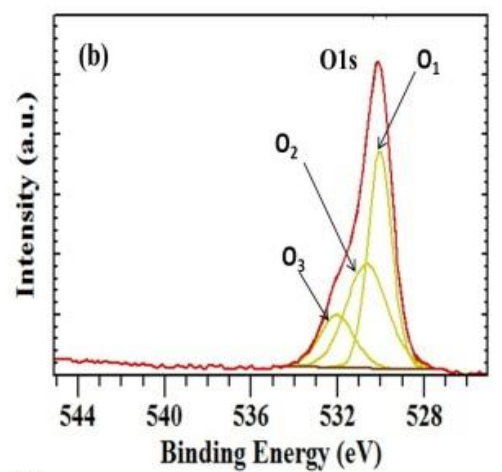

(b)

Fig. 4: XPS spectra of (a) pure $\mathrm{ZnO}$ and (b) $0.05 \%$ Pt doped $\mathrm{ZnO}$.

\subsection{Gas Sensing Properties}

The sensing response of undoped and $\mathrm{Pt}$-doped $\mathrm{ZnO}$ gas sensor for $\mathrm{H}_{2}$ gas concentration of $250 \mathrm{ppm}$ was measured at different operating temperatures and results are shown in Fig. 5. The optimum operating temperature for pure $\mathrm{ZnO}$ and $\mathrm{Pt}$ doped $\mathrm{ZnO}$ are found to be 400 and $350^{\circ} \mathrm{C}$ respectively. The semiconductor metal oxide gas sensing phenomena involves change in electrical conductance due to the gas adsorption and desorption on the surface of sensor material. Metal oxide sensor on exposure to air, adsorbs the atmospheric oxygen on its surface which gets converted to $\mathrm{O}^{-}, \mathrm{O}^{2-}$ or $\mathrm{O}_{2}^{-}$ion by capturing an electron from the conduction band. On introduction of $\mathrm{H}_{2}$ gas, the oxygen reacts with $\mathrm{H}_{2}$ forming $\mathrm{H}_{2} \mathrm{O}$ and thereby releasing electrons to conduction band.

Figure 6 exhibits the sensor response versus time plots for pure and $0.05 \% \mathrm{Pt}$ doped $\mathrm{ZnO}$. The enhanced sensor response of $\mathrm{Pt}$ doped $\mathrm{ZnO}$ is explained by escalation of surface reaction through spill over effect. According to this effect the target gas molecules adsorbed at the metal cluster dissociates and the atoms spill over onto the metal oxide surface. On adsorption of reducing gas, these ions may interact with adsorbed or lattice surface oxygen molecules and alter the SMO's conductance. Another plausible reason is the increase in defect (oxygen vacancies) due to dopant incorporation in $\mathrm{ZnO}$ lattice which is supported by Raman studies.

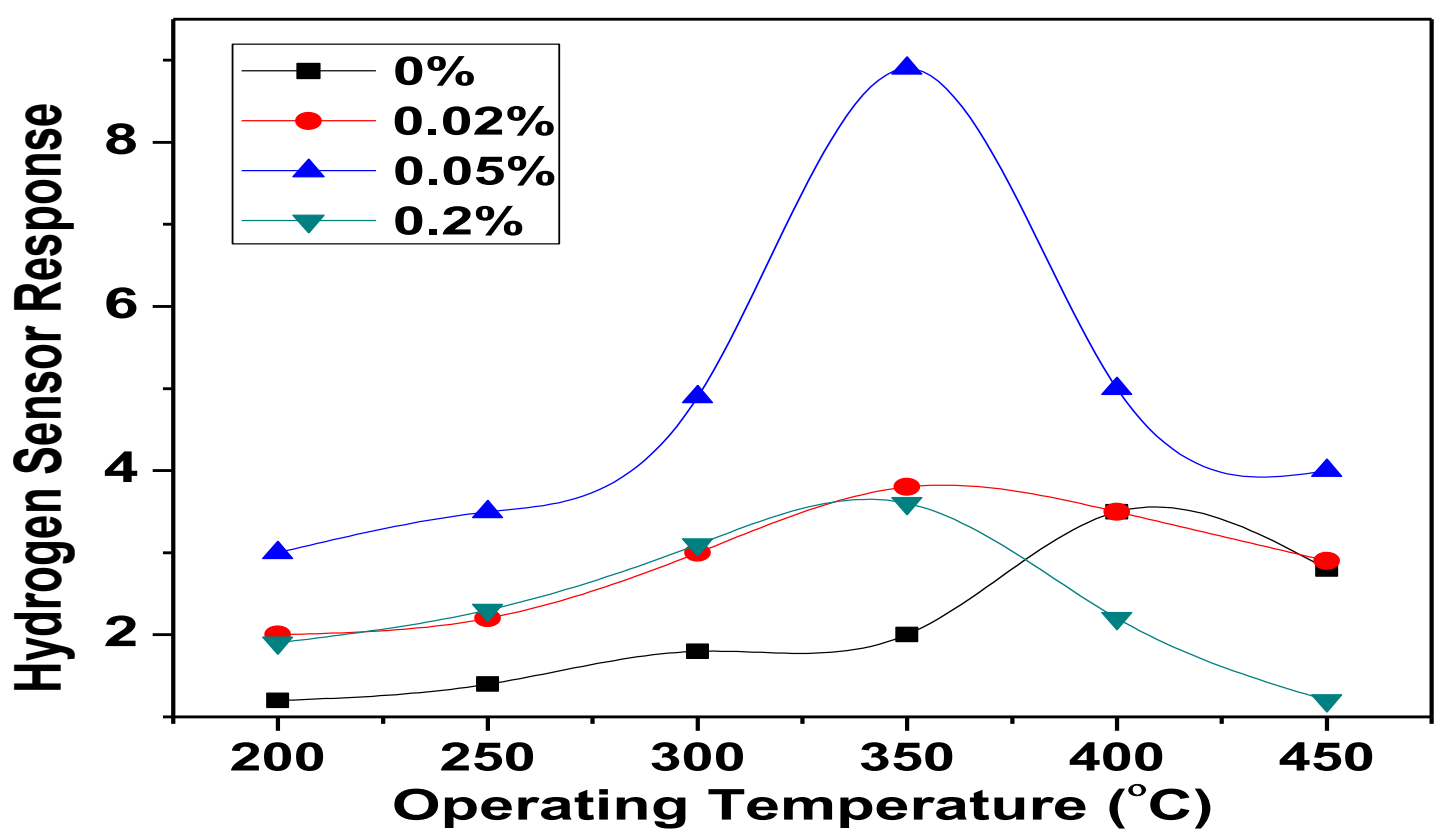

Fig. 5: Variation of $\mathrm{H}_{2}$ sensor response of (a) pure, (b) $0.02 \% \mathrm{Pt}$ doped, (c) $0.05 \% \mathrm{Pt}$ doped and (d) $0.2 \% \mathrm{Pt}$ doped $\mathrm{ZnO}$ with operating temperature. 


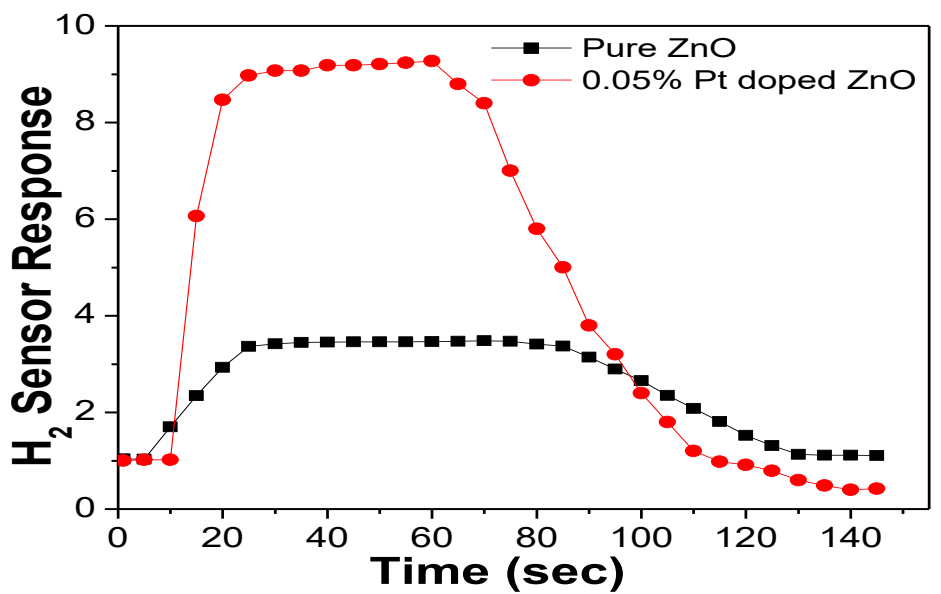

Fig. 6: Variation of $\mathrm{H}_{2}$ sensor response versus time plot of pure and $0.05 \% \mathrm{Pt}$ doped $\mathrm{ZnO}$.

\section{Conclusion}

Pure and Pt doped $\mathrm{ZnO}$ nanostructures were successfully synthesized by co-precipitation route. XRD studies confirm the hexagonal wurtzite structure of prepared samples and incorporation of $\mathrm{Pt}$ in $\mathrm{ZnO}$ in case of doped samples. Raman spectroscopy and XPS analysis signify increase in oxygen vacancies with the addition of dopants. FESEM studies reveal that with addition of dopants the growth of nanoparticles in a particular direction is inhibited. The addition of Pt has lowered the optimum operable temperature from 400 to $350^{\circ} \mathrm{C}$ and has significantly improved the sensor response of $\mathrm{ZnO}$ towards hydrogen vapour due to change in morphology, increase in oxygen vacancies and catalytic nature of Pt.

\section{Acknowledgements}

Anita Hastir would like to acknowledge financial support from the INSPIRE Fellowship, Department of Science \& Technology, India. Thanks to UGC-UPE, India and DST-FIST, India for providing instrumental facilities. We would also like to thank Prof. Robert L. Opila at the University of Delaware for his help in providing XPS facilities.

\section{References}

[1] Y. Irokawa, "Hydrogen Sensors Using Nitride-Based Semiconductor Diodes: The Role of Metal/Semiconductor Interfaces," Sensors, vol. 11, pp. 674-695, 2011.

[2] Y. Shen, W. Wang, A. Fan, D. Wei, W. Liu, C. Han, Y. Shen, D. Meng, and X. San, "Highly sensitive hydrogen sensors based on $\mathrm{SnO}_{2}$ nanomaterials with different morphologies," Int. J. Hydrogen Energ., vol. 40, pp. 15773-15779, 2015.

[3] Y. Lim, Y. Lee, J. Heo, and H. Shin, "Highly sensitive hydrogen gas sensor based on a suspended palladium/carbon nanowire fabricated via batch microfabrication processes," Sens. Actuators B, vol. 210, pp. 218-224, 2015.

[4] Z. L. Wang, "Zinc oxide nanostructures: growth, properties and applications," J. Phys. Condens. Mat., vol. 16, pp. 829-858, 2004.

[5] V. K. Jayaraman, A. M. Alvarez, and M. L. O. Amador, "A simple and cost-effective zinc oxide thin film sensor for propane gas detection," Mater. Lett., vol. 157, pp. 169-171, 2015.

[6] N. Kohli, O. Singh, and R. C. Singh, "Influence of $\mathrm{pH}$ on particle size and sensing response of chemically synthesized chromium oxide nanoparticles to alcohols," Sens. Actuators B, vol. 158, pp. 259-264, 2011.

[7] C. Sronsri, P. Noisong, and C. Danvirutai, "Synthesis, characterization and vibrational spectroscopic study of Co, Mg co-doped LiMnPO 4 ," Spectrochim. Acta A, vol. 153, pp. 436-444, 2016.

[8] J. P. Singh, R. C. Srivastava, H. M. Agrawal, and R. Kumar, "Micro-Raman investigation of nanosized zinc ferrite: effect of crystallite size and fluence of irradiation," J. Raman Spectrosc., vol. 42, pp. 1510-1517, 2011.

[9] A. K. Arora, M. Rajalakshmi, T. R. Ravindran, and V. Sivasubramanian, "Raman spectroscopy of optical phonon confinement in nanostructured materials," J. Raman Spectrosc., vol. 38, pp. 604- 617, 2007.

[10] Y.C. Liang, H.Y. Hsia, Y.R. Cheng, C.M. Lee, S.L. Liu, T. Yin and C.C. Chung, "Crystalline quality-dependent gas detection behaviors of zinc oxide-zinc chromite p-n heterostructures," Cryst. Eng. Comm., vol. 17, pp. 4190-4199, 2015. 